\title{
Clasificación de clorosis en hojas de árboles de naranja mediante aprendizaje automático
}

\author{
Juan P-Salazar, Eddy Sánchez-DelaCruz, R.R. Biswal \\ Instituto Tecnológico Superior de Misantla, Posgrado en Sistemas Computacionales, \\ Misantla, Veracruz, \\ México \\ jpsalazar1122@gmail.com, \{esanchezd,rroshanb $\} @$ itsm.com.mx
}

\begin{abstract}
Resumen. La fruta de naranja es considerada como uno de los principales productos agroalimentarios de mayor producción en México [13]. El volumen promedio producido es de 4.2 millones de toneladas anuales y el estado de Veracruz a nivel nacional es el primer productor aportando el $44.5 \%$ del volumen total [12]. La producción eficiente de este fruto se ve afectada por la diseminación de enfermedades en los campos de producción y es la principal causa de pérdidas económicas. Para solucionar esta problemática se han implementado técnicas computacionales con la capacidad de clasificar e identificar dichas enfermedades. Esta investigación propone evaluar un conjunto de clasificadores para su implementación en la detección de enfermedades usando características sintomáticas en hojas de árboles de naranja Valencia (Citrus Sinensis). Las técnicas y métodos de procesamiento de imágenes para la creación de una base de datos y clasificación binaria aquí aplicados, nos llevaron a encontrar que tres clasificadores ensamblados (metaclasificadores) en combinación con el algoritmo de aprendizaje profundo perceptrón multicapa (D14jMlp: Deep Learning for java with Multilayer Perceptron) tienen la capacidad de lograr una clasificación del $100 \%$ en bases de datos de tamaño pequeño y mejoradas alcanzado por la aplicación de técnicas de procesamiento de imágenes y minería de datos.
\end{abstract}

Palabras clave: Técnicas computacionales, procesamiento de imágenes, algoritmos ensamblados, aprendizaje profundo, perceptrón multicapa, clasificación.

\section{Classification of Chlorosis in Leaves of Orange Trees Using Machine Learning}

\begin{abstract}
Oranges are considered to be one of the main agroalimentary products of major production in México [13]. The average volume produced is 4.2 million tons and Veracruz turns out to be the first contributing state with $44.5 \%$ of the total volume [12]. Efficient production of this fruit is affected by the spread of diseases in production fields resulting in major economic losses. To solve this problem, computer techniques have been implemented with the main objective to classify and identify these diseases. This research proposes to evaluate various
\end{abstract}




\begin{abstract}
classifiers for implementation in disease detection using symptomatic features in leaves of Valencia orange trees (Citrus Sinensis). The procedures and methods for the creation of a database and binary classification applied, led us to find out that three Metalearning Algorithms in combination with the D14jMlp (Deep Learning for java with Multilayer Perceptron) have the ability to accomplish a classification of $100 \%$ in small dimension and improved databases achieved by the application of image processing and data mining techniques.
\end{abstract}

Keywords: Computer techniques, image processing, metalearning algorithms, deep learning, multilayer perceptron, classification.

\title{
1. Introducción
}

La detección oportuna de enfermedades en cultivos es un factor determinante en una eficiente producción agrícola, por lo que es esencial la identificación de manera temprana. En la actualidad aunque el avance tecnológico aplicado al área agrícola crece de manera exponencial aún existe rezago en el estudio de muchas enfermedades. La implementación de herramientas computacionales para la clasificación se ha dirigido con gran intensidad a Huanglongbing (HLB), sin embargo, existen enfermedades a las cuales no se le ha dado importancia relevante como el caso de Clorosis Variegada de los Cítricos (CVC) y Virus Psorosis de los Cítricos (CPsV), estas enfermedades son consideradas destructivas y de peligro para la producción de cítricos y son comparadas con HLB por el impacto en pérdidas económicas [1]. Los síntomas visibles en las hojas de las plantas son los primeros que alertan sobre una posible plaga o alguna deficiencia nutrimental. El CVC al igual que el HLB, el CPsV y la deficiencia de Zinc se caracterizan por la decoloración de las hojas de cítricos [4], debido a esta similitud, es difícil confiar solo en los síntomas foliares para la identificación. Para solucionar esta problemática se han implementado técnicas como análisis y diferenciación fotoquímica entre HLB y deficiencia de Zinc [2], espectroscopia de infrarrojo medio para la detección de HLB [1], sensores de visión [10] y métodos de visión por computadora, procesamiento de imágenes y minería de datos avanzada empleando algoritmos para la clasificación de enfermedades de cítricos [11], técnicas aplicables a la clasificación de enfermedades mediante la presencia de características foliares como la clorosis.

Cualquier tipo de clorosis visible en las hojas de cítricos precisa de ser investigada. La pérdida de color verde es una indicación importante de problemas de crecimiento y pérdidas de productividad; los causantes pueden ser tanto bacterias, virus o deficiencias nutricionales. La CVC es causada por el agente "Xylella fastidiosa" [14]. La bacteria vive y se multiplica en la savia de las plantas de cítricos, bloqueando la absorción de agua. Los síntomas foliares del CVC son muy similares a la deficiencia de nutrientes y otras enfermedades como HLB y CPsV. Los primeros síntomas de la hoja se parecen a la deficiencia de Zinc con áreas cloróticas intervenales en la superficie superior, las hojas pueden ser más pequeñas de lo normal y los síntomas de la hoja como la decoloración de las áreas afectadas y las manchas obscuras o marrón con acumulación de gomosis en el envés se intensifican mucho más en hojas maduras, en la última etapa las manchas comienzan a extenderse hacia el borde y el tejido se seca [4,8]. Estas características sintomáticas de las hojas se pueden aprovechar para la clasificación de enfermedades empleando técnicas de procesamiento de imágenes, minería de datos y 


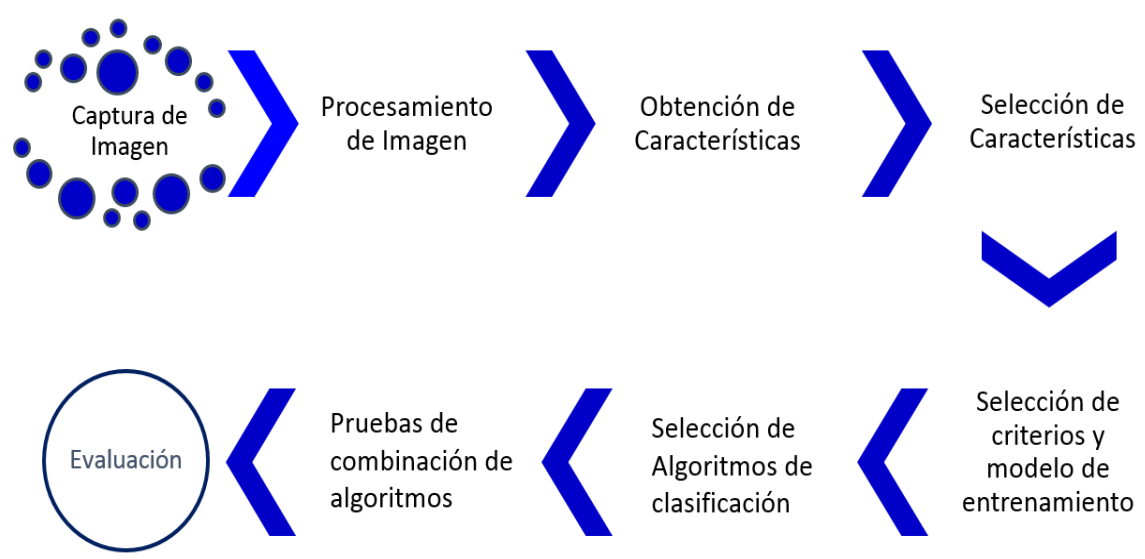

Fig. 1. Metodología propuesta.

aprendizaje profundo con la finalidad de obtener un clasificador eficientemente capaz de identificar las diferencias entre la presencia sintomática de clorosis y hojas visiblemente sanas.

\subsection{Propuesta de solución}

Esta investigación propone un método de procesamiento de imágenes [15] y minería de datos para la clasificación binaria de presencia sintomática de clorosis en hojas de naranja y hojas visiblemente sanas para la obtención de un clasificador eficiente. El diagrama siguiente proporciona una breve descripción de la metodología aplicada, la cual se describe a detalle en la sección cuatro (ver Figura 1).

\section{Trabajos previos}

Pourreza et al., en una de sus investigaciones desarrollaron un sensor de visión aprovechando la rotación del plano de polarización de la luz en algunas longitudes de onda para la identificación de síntomas de HLB; los resultados confirmaron que la iluminación polarizada de banda estrecha a $591 \mathrm{~nm}$ aumentó significativamente la precisión del diagnóstico en la clasificación y detección de HBL [10].

Camponez et al., aplicaron un método de diagnóstico para la detección de presencia de HLB y CVC en hojas de árboles de naranja dulce mediante espectroscopia infrarroja con reflectancia total atenuada de la transformada de Fourier y el clasificador inducido mediante regresión parcial de mínimos cuadrados. El modelo consideró cuatro clases de hojas, una con hojas saludables, con CVC sintomático, con HLB sintomático y con HBL asintomático en donde los resultados obtenidos arrojaron un porcentaje del $93.8 \%$ en la correcta identificación de los cuatro tipos de hojas estudiados [1].

Pydipati et al., propusieron un modelo empleando un método de co-ocurrencia (CCM) para determinar si el tono de color, la saturación y la intensidad (HSI) basados 
en características de textura en conjunción con algoritmos de clasificación estadística podrían ser utilizados para identificar las hojas de cítricos enfermas y normales bajo condiciones de laboratorio. Se evaluaron muestras de hojas de cítricos normales y enfermas estas últimas con puntos grasos, melanosis y escamosis. El análisis discriminante de la muestra de hojas con características de textura CCM logró precisiones de clasificación de más del $95 \%$ para todas las clases cuando se utilizaron la tonalidad de color y la saturación de las características de textura [11].

Ceballos et al., desarrollaron y una técnica optimizada basada en el método GC-MS para el análisis de metabolitos no alcanzables en hojas de cítricos. Las muestras de HLB y deficiencia de zinc de hojas de árboles de naranja dulce fueron sometidas al proceso de micro extracción en fase sólida y tratamientos de derivatización anteriores al análisis GC-MS. El análisis de componentes principales alcanzó una correcta clasificación de todos los derivativos de extractos líquidos empleando biomarcadores para la rápida diferenciación de HLB y deficiencia de zinc [2].

Sankaran et al., muestran en su investigación un enfoque en la aplicación de espectroscopia en el infrarrojo medio para la detección de HLB. Se emplearon muestras de hojas saludables, con HLB y deficiencia de nutrientes en árboles mediante dos formas de procesamiento y analizándolas usando un espectrómetro portable y robusto. Se utilizaron datos de la absorbancia espectral en el rango de los 5.15-10.72nm y se procesaron mediante corrección de alineamiento básica, corrección de datos atípicos y remoción de datos de las longitudes de banda de la absorbancia del agua. El primero y el segundo proceso derivativo fueron calculados mediante el método de SavitzkyGolay y analizados mediante análisis de componentes principales y los records de este fueron clasificados usando el algoritmo de análisis discriminante cuadrático (QDA) y el algoritmo de vecinos más cercanos $(\mathrm{KNN})$, determinando que el algoritmo KNN obtuvo por arriba de $95 \%$ en comparación con el algoritmo QDA, en este caso los datos analizados en bruto proporcionaron mejor precisión en la clasificación comparados con los datos derivativos del procesamiento uno y dos [12].

Mohanty et al., trabajaron en el desarrollo de un clasificador de imágenes de alta precisión con la finalidad de diagnosticar enfermedades de plantas empleando una base de datos del proyecto de recolección de hojas con presencia de enfermedades y saludables llamado PlantVillage del cual se emplearon 54,306 imágenes en la clasificación de 26 enfermedades distintas de 16 especies de cultivos mediante el enfoque de una red neuronal convolucional. El desempeño de los modelos de clasificación fue medido basado en su habilidad de predecir los pares correctos cultivoenfermedad, dadas 38 posibles clases. El modelo con mejor desempeño alcanzo un puntaje medio F1 de 0.9934 es decir una precisión del $99.35 \%$ demostrando la factibilidad técnica de este enfoque [9].

Kadir et al., propusieron un método que captura información de color como un aspecto importante para la identificación de enfermedades en hojas. En esta investigación se incorporaron características de forma geométrica, color y textura de cada hoja; empleando una Red Neuronal Probabilística como clasificador. Lo resultados experimentales mostraron que el método de clasificación elegido obtuvo una precisión media del $93.75 \%$ cuando fue probado con la base de datos Flavia la cual contiene 32 tipos de hojas de plantas [7]. 

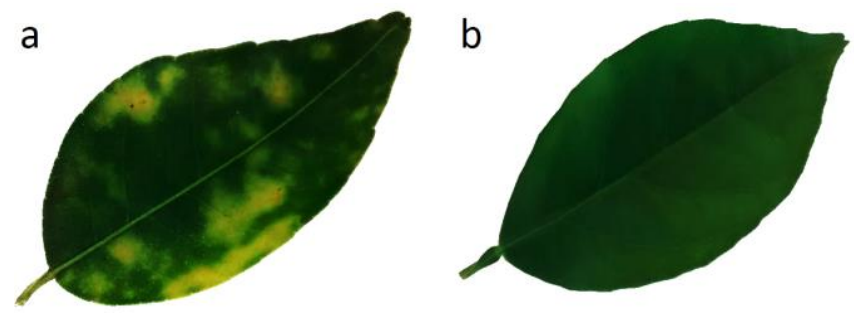

Fig. 2. Muestras de hojas (a) con presencia de clorosis y (b) saludable.
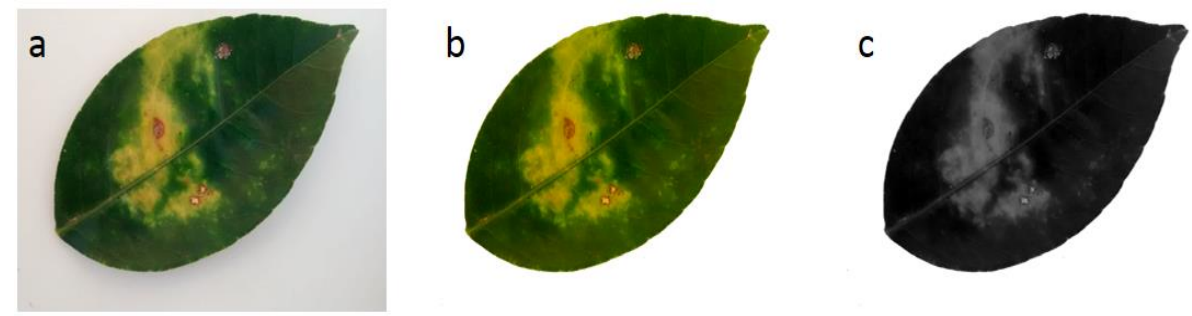

Fig. 3. Procesamiento de imágenes (a) original (b) segmentada y mejorada (c) escala de grises.

\section{Materiales y método}

\subsection{Adquisición de la base de datos}

Se recolectaron hojas enfermas con presencia de clorosis y hojas saludables de árboles de naranja dulce Valencia (Citrus Sinensis), se tomaron fotografías individuales en un ambiente controlado para reducir el brillo y reflectancia en las imágenes, se empleó la cámara de un dispositivo móvil con calidad de 5 megapíxeles y una resolución de 2592x1456; las fotografías obtenidas se recopilaron en una base de datos, 60 imágenes de hojas saludables y 60 con clorosis sintomática (ver Figura 2.).

La obtención de características de las imágenes de hojas se realizó mediante algoritmos de reconocimiento de patrones aplicados a procesamiento digital de imágenes [15]. El primer paso fue la eliminación del brillo residual y una mejora de contraste mediante la ecualización del histograma, el siguiente fue realizar una segmentación para eliminar el fondo de la imagen empleando umbralización con el método de otsu, aprovechando la diferencia de los niveles de intensidad de las hojas y el fondo, esto nos dio como resultado la obtención de nuestra región de interés que es solo la hoja, posteriormente la imagen se convirtió a escala de niveles de gris para la obtención de los valores de cada pixel y características estadísticas estándar del histograma de frecuencias. Estos valores se guardaron en un archivo en formato CSV para la generación de nuestra base de datos (ver Figura 3.).

La base de datos obtenida contiene la información de las características más relevantes de las hojas, en primer término, las dos clases se distribuyen en forma de filas, 60 de ellas son nombradas saludable y 60 enferma, la primera para las hojas 
Juan P-Salazar, Eddy Sánchez-DelaCruz, R.R. Biswal

Tabla 1. Resumen de las características de la base de datos obtenida.

\begin{tabular}{cccc}
\hline Clase & Categoría & $\begin{array}{c}\text { Características de } \\
\text { Niveles de Gris }\end{array}$ & $\begin{array}{c}\text { Número de } \\
\text { Características }\end{array}$ \\
\hline 1 & Saludable & Valores de Pixeles & 256 \\
\hline 1 & Saludable & Características Estadísticas & 6 \\
\hline 2 & Enferma & Valores de Pixeles & 256 \\
\hline 2 & Enferma & Características Estadísticas & 6 \\
\hline & & Total de Características por Clase & 262 \\
\hline
\end{tabular}

Tabla 2. Conjuntos de entrenamiento y prueba.

\begin{tabular}{cccc}
\hline Clase & Entrenamiento & Prueba & Total \\
\hline Saludable & 40 & 20 & 60 \\
Enferma & 40 & 20 & 60 \\
Total & 80 & 40 & 120 \\
\hline
\end{tabular}

visiblemente saludables y la segunda para las hojas con presencia sintomática de clorosis respectivamente.

Cada columna de la base de datos contiene cada uno de los atributos de cada hoja iniciando con los valores de cada pixel del histograma de frecuencia de niveles de gris desde 0 hasta 255, es decir ocupa las primeras 256 columnas; las ultimas 6 columnas son ocupadas por las características estadísticas del histograma de frecuencia incluyendo la media, desviación estándar, curtosis, oblicuidad (índice de asimetría), media laplaciana y gradiente límite de la media [15]. Por último los nombres de las dos clases: saludable y enferma, se ubicaron en la última columna con el propósito evitar errores en su procesamiento. La tabla siguiente brinda una breve descripción de las características de la base de datos (ver Tabla 1).

\subsection{Tratamiento de los datos}

El procesamiento de la base de datos se realizó mediante una combinación de algoritmos ensamblados con el algoritmo de aprendizaje profundo perceptrón multicapa (D14jMlp). Para la realización de las pruebas se emplearon 3 criterios de evaluación el primero por validación cruzada, el segundo empleando una división de porcentaje $2 / 3$ para entrenamiento y $1 / 3$ para pruebas y por último por muestra reprentativa calculada de 92 instancias con un porcentaje de $23.33 \%$ del total de instancias. El procedimiento consistió en enfrentar todos los algoritmos metaclasificadores contra el algoritmo clasificador D14jMlp y evaluar el porcentaje de precisión que obtiene cada combinación, estos resultados se pueden apreciar en la sección 4. El criterio de división de porcentaje de $2 / 3$ para entrenamiento y $1 / 3$ para pruebas, con el que se obtuvo el valor óptimo, se muestra en la Tabla 2.

Los algoritmos utilizados para realizar la combinación con el algoritmo D14jMlp (Deep Learning for Java with Multilayer Perceptron) se categorizan dentro de los Algoritmos Metaclasificadores. 


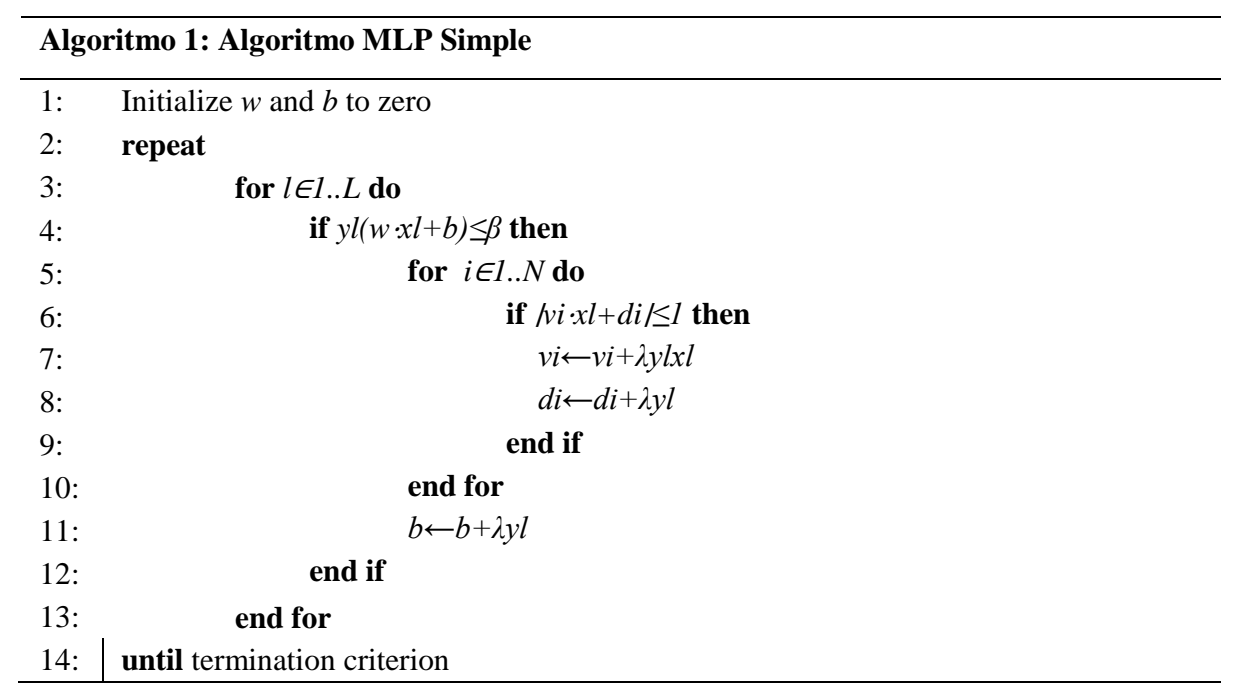

Tabla 3. Resultados de la combinación de algoritmos metaclasificadores y D14jMlp.

\begin{tabular}{|c|c|c|c|c|}
\hline Metaclasificadores & $\begin{array}{l}\text { Aprendizaje } \\
\text { Profundo }\end{array}$ & $\begin{array}{l}\text { Val. Cruzada } \\
\text { 10iteraciones }\end{array}$ & $2 / 3-1 / 3$ & $\begin{array}{c}\text { Muestra } \\
\text { Representativa }\end{array}$ \\
\hline ---------- & Dl4jMlp & 97.5 & 97.5 & 92.2222 \\
\hline AttributeSelectedClassifier & Dl4jMlp & 99.1667 & 100 & 98.8889 \\
\hline Bagging & D14jMlp & 50 & 42.5 & 44.4444 \\
\hline ClassificationViaClustering & Dl4jMlp & 62.5 & 67.5 & \\
\hline ClassificationViaRegression & Dl4jMlp & 50 & 42.5 & 44.4444 \\
\hline CVParameterelection & Dl4jMlp & 50 & 42.5 & 44.4444 \\
\hline FilteredClassifier & Dl4jMlp & 99.1667 & 100 & 96.6667 \\
\hline LogitBoost & Dl4jMlp & 50 & 57.5 & 55.5556 \\
\hline MultiClassClasifier & Dl4jMlp & 50 & 42.5 & 44.4444 \\
\hline MultiScheme & Dl4jMlp & 50 & 42.5 & \\
\hline MultiSearch & Dl4jMlp & 50 & 42.5 & 44.4444 \\
\hline OrdinalClassClassifier & Dl4jMlp & 97.5 & 97.5 & 92.2222 \\
\hline RandomCommite & Dl4jMlp & 50 & 42.5 & 44.4444 \\
\hline RandomizableFilteredClassif & Dl4jMlp & 54.1667 & 60 & 51.1111 \\
\hline RandomSubSpace & Dl4jMlp & 96.6667 & 100 & 98.8889 \\
\hline Stacking & Dl4jMlp & 50 & 57.5 & 44.4444 \\
\hline ThresholdSelector & Dl4jMlp & 54 & 42.5 & 55.5556 \\
\hline Vote & Dl4jMlp & 50 & & \\
\hline WeightedInstancesHandlerW & Dl4jMlp & 50 & 42.5 & 44.4444 \\
\hline
\end{tabular}

La función de los Algoritmos Metaclasificadores consiste en mejorar el aprendizaje de los clasificadores convirtiéndolos en aprendices más poderosos. En los resultados, 
los mejores clasificadores fueron tres, cada uno con un porcentaje perfecto de clasificación, a continuación se hace una descripción resumida del funcionamiento de estos algoritmos [6].

AttributeSelectedClassifier: Se encarga de seleccionar atributos reduciendo la dimensionalidad de los datos para eliminar datos redundantes y aplicar un clasificador al resultado del conjunto de datos obtenido; esto permite el uso de un método de selección de atributos y un algoritmo de aprendizaje específico como parte de un esquema de clasificación, asegurando que el conjunto de atributos elegidos es seleccionado solamente basado en los datos de entrenamiento y evaluado en los datos de prueba.

FilteredClassifier: Este algoritmo se encarga de aplicar un filtro al conjunto de datos antes de aplicar un algoritmo de aprendizaje, filtrando los datos de entrenamiento sin filtrar los de prueba además emplea un clasificador el cual usa los datos previamente filtrados con la finalidad de reducir el conjunto de datos a solamente los de mayor importancia. Este algoritmo se utiliza para la evaluación equitativa de discretización supervisada esto lo realiza solo en los datos de prueba usando intervalos de discretización computados para los datos de entrenamiento que en la práctica equivale al procesamiento de datos completamente frescos.

RandomSubSpace: Construye un ensamble de clasificadores, cada uno entrenado usando un subconjunto seleccionado aleatoriamente de atributos de entrada. Aparte del número de iteraciones y la inserción aleatoria usada, provee un parámetro de control del tamaño del subconjunto de atributos, reduciendo el tamaño de los conjuntos de datos para una eficiente clasificación.

El algoritmo Dl4jMlp se expresa como ecuación matemática de una forma razonablemente natural, este algoritmo tiene como base la función de regresión y clasificación mediante perceptrón multicapa, este algoritmo se explica de manera resumida a continuación [6].

Perceptrón Multicapa también llamado red neuronal de retroalimentación, es un modelo de aprendizaje profundo por excelencia, el objetivo de este algoritmo es aproximar una función $f^{*}$. Un ejemplo claro para un clasificador $y=f^{*}(x)$ mapea un valor de $x$ a una categoría $y$. Una red de retroalimentación define un mapeo $y=f(x, \theta)$ y aprende los valores del parámetro $\theta$ el cual resulta en la mejor función de aproximación. En este algoritmo la información fluye a través de la función que ha sido evaluada desde $x$ a través del cómputo intermediario para definir $f$ hasta llegar a la salida $y$ [5]. Esta clase de red neuronal se compone de una capa de entrada, capas ocultas intermedias y una capa de salida, en donde cada conexión tiene una ponderación (peso) y cada nodo realiza una suma ponderada de sus entradas, umbralizando el resultado; no contiene ningún ciclo y la salida de la red depende solamente de las instancias actuales de entrada; su ventaja es la capacidad de aprender a ignorar atributos irrelevantes y representa límites no lineares de decisión. El proceso de operación de este algoritmo se muestra a continuación [3].

\section{Experimentos y resultados}

El análisis realizado aplicando todos los Algoritmos Metaclasificadores en combinación con el algoritmo D14jMlp, arrojó los mejores resultados con los criterios 
Clasificación de clorosis en hojas de árboles de naranja mediante aprendizaje automático

Tabla 4. Matriz de confusión para los tres pares de algoritmos.

\begin{tabular}{ccc}
\hline $\mathbf{a}$ & $\mathbf{b}$ & Clasificadas como \\
\hline $\mathbf{2 3}$ & 0 & $\mathrm{a}=$ Saludables \\
\hline 0 & 17 & $\mathrm{~b}=$ Enfermas \\
\hline
\end{tabular}

de división de $2 / 3$ - $1 / 3$ logrados por tres metaclasificadores, AttributeSelectedClassifier, FilteredClassifier y RandomSubSpace, todos con una precisión de clasificación del $100 \%$. Las combinaciones completas y sus resultados se muestran en la Tabla 3.

Los experimentos se realizaron en un ordenador con: OS Windows 8, Intel(R) Core(TM) i3-2330M CPU 2.20 GHz, RAM 8.00 GB, HD 320 GB, sistema operativo de 64 bits y procesador x64. Además, para el procesamiento de imágenes y la ejecución de los algoritmos descritos en esta sección, utilizamos el lenguaje de programación MATLAB ${ }^{\circledR}$ y el software de minería de datos WEKA v.3.8 respectivamente.

\section{Análisis y discusión}

El porcentaje alcanzado de instancias clasificadas correctamente por los tres algoritmos metaclasificadores con D14jMlp se debe principalmente al tratamiento de datos realizado por cada clasificador, como se explica a continuación:

- El algoritmo AttributeSelectedClassifier realiza una reducción de la dimensionalidad de los datos de entrenamiento al seleccionar los atributos antes de pasarlos al clasificador.

- El algoritmo clasificador FilteredClassifier pasa previamente los datos por un filtro arbitrario antes de ser clasificados.

- El algoritmo RandomSubSpace construye una clasificación basada en un árbol de decisión la cual logra mantener una alta precisión en el entrenamiento de datos, mejorando la precisión cuanto más crece la complejidad de los datos.

- El clasificador Dl4jMlp realiza clasificaciones y regresiones ignorando atributos irrelevantes.

Los resultados obtenidos de las tres combinaciones, se representaron en una matriz de confusión para cada par respectivo cabe aclarar que en los tres casos se obtuvo la misma matriz de confusión, esta se muestra en Tabla 4.

En la matriz de confusión del clasificador AttributeSelectedClassifier + Dl4jMlp, FilteredClassifie $+D l 4 j M l p$, así como RandomSpace $+D l 4 j M l p$, se puede apreciar que a pesar de que hubo una reducción en el número de instancias para clasificar no se obtuvieron instancias clasificadas erróneamente. Es decir, los valores 23 y 17 son los casos de hojas saludables y enfermas clasificadas correctamente, mientras que las casillas con el valor 0 indican que debido a la reducción de datos hay una reducción completa de la confusión en la clasificación de dichas instancias. 


\section{Conclusiones y trabajo futuro}

Al combinar AttributeSelectedClassifier+Dl4jMlp, FilteredClassifie + Dl4jMlp y RandomSpace $+D l 4 j M l p$, se obtuvo un porcentaje perfecto de clasificación en comparación con los métodos aplicados en las investigaciones del estado del arte en donde el porcentaje mejor obtenido fue de $99.35 \%$. La obtención del $100 \%$ de instancias clasificadas correctamente se debió a la combinación de diferentes factores, desde el tamaño ligero de la base de datos, el procesamiento digital de las imágenes, la combinación de algoritmos de aprendizaje automático, el tratamiento de clasificación binaria y sobre todo la reducción de dimensionalidad, además del filtrado de datos aplicado a la base de datos por los clasificadores combinados.

Para la continuación futura de esta investigación se propone realizar las siguientes acciones:

- Aplicar la combinación de algoritmos obtenida de este estudio a una clasificación multiclase, agregando más enfermedades, además de características geométricas y de Haralick.

- Realizar una selección de mejores atributos, para encontrar la combinación ideal de algoritmos para realizar una comparación de resultados con todos los atributos y con los mejores.

- Implementar las técnicas usadas en esta investigación en una herramienta de detección para mejorar la precisión en la identificación de enfermedades.

\section{Referencias}

1. Camponez, M., Boasa, P., Miloria, D., Ferreiraa, E., Silva, M., Machadoc, M., Belleted, B., Fernandes-Da Silva, M.: Infrared spectroscopy: A potential tool in huanglongbing and citrus variegated chlorosis diagnosis. Talanta 91(1), pp. 1-6 (2012)

2. Cevallos, J., García-Torres, R., Etxeberria, E., Reyes-De Corcuera, J.: Gc-ms analysis of headspace and liquid extracts for metabolomic diferentiation of citrus huanglongbing and zinc defciency in leaves of Valencia sweet orange from commercial groves. Phytochemical Analysis, pp. 236-246 (2010)

3. Collobert, R., Bengio, S.: Links between perceptrons, MLPs and SVMs. In: Proceedings of the twenty-first international conference on Machine learning 23 (ACM) (2004)

4. Citrus variegated chlorosis (CVC): http://idtools.org/id/citrus/diseases/factsheet .php?name=Citrus+variegated+chlorosis $+\% 28 \mathrm{CVC} \% 29$ (2018)

5. Goodfeloow, I., Bengio, Y., Courville, A.: Deep Learning, pp. 167 (2016)

6. Witten, I., Frank, E., Hall, M.: Data Mining, pp. 234-582, Morgan Kaufmann (2011)

7. Kadir, A., Nugroho, L., Susanto, A., Santosa, P.: Leaf classification using shape, color, and texture features. International Journal of Computer Trends and Technology (2011)

8. Citrus variegated chlorosis (xylella fastidiosa): http://www.padil.gov.au/pests-anddiseases/pest/main/136652 (2018)

9. Mohanty, S., Hughes, D., Salathe, M.: Using deep learning for image-based plant disease detection. 4 Frontiers in Plant Science (2010)

10. Pourreza, A., Lee, W., Etxeberria, E., Banerjee, A.: An evaluation of a vision-based sensor performance in huanglongbing disease identfication. Biosystems Engineering, 130(1), pp. 13-22 (2015) 
11. Pydipati, R., Burks, T., Lee, W.: Identification of citrus disease using color texture features and discriminant analysis. Computers and Electronics in Agriculture, 52(2), pp. 4959 (2006)

12. Sankaran, S., Ehsani, R., Etxeberria, E.: Mid-infrared spectroscopy for detection of huanglongbing (greening) in citrus leaves. Talanta, 83(3), pp. 574-581 (2010)

13. Secretaría de Agricultura, Ganadería, Desarrollo Rural, Pesca y Alimentación (SAGARPA): Se consolida México como quinto productor mundial de naranja (2018)

14. Servicio Nacional de Sanidad Inocuidad y Calidad Agroalimentaria: Plagas reglamentadas de los cítricos (X. fastidiosa subsp. pauca, X. citri subsp. citri, G. citricarpa y CLVc) (2018)

15. Da-Wen, S.: Computer Vision Technology for Food Quality Evaluation. Nikki Levy (2016) 